\title{
Choosing the right survey-patient reported outcomes in esophageal surgery
}

\author{
Maira Ahmed ${ }^{1 \#}$, Angus Lau ${ }^{1 \#}$, Dhruvin H. Hirpara ${ }^{2}$, Biniam Kidane ${ }^{1,3,4}$ \\ ${ }^{1}$ College of Medicine, University of Manitoba, Winnipeg, Manitoba, Canada; ${ }^{2}$ Department of Surgery, University of Toronto, Toronto, Ontario, \\ Canada; ${ }^{3}$ Section of Thoracic Surgery, Health Sciences Centre, Winnipeg, Manitoba, Canada; ${ }^{4}$ Research Institute in Oncology and Hematology, \\ Cancer Care Manitoba, Manitoba, Canada \\ Contributions: (I) Conception and design: B Kidane; (II) Administrative support: B Kidane; (III) Provision of study materials or patients: None; (IV) \\ Collection and assembly of data: All authors; (V) Data analysis and interpretation: M Ahmed, A Lau, DH Hirpara; (VI) Manuscript writing: All \\ authors; (VII) Final approval of manuscript: All authors. \\ \#These authors contributed equally to this work. \\ Correspondence to: Biniam Kidane, MD, MSc, FRCSC. GH604-820 Sherbrook Street, Winnipeg, Manitoba R3A 1R9, Canada. \\ Email: bkidane@hsc.mb.ca.
}

\begin{abstract}
Patient reported outcomes (PROs) fulfill a crucial and unique niche in patient management, providing health-care providers a glimpse into their patients' health experience. This is of utmost importance in patients with benign and malignant disorders of esophagus requiring surgery, which carries significant morbidity, in part due to a high burden of symptoms affecting health-related quality of life (HRQOL). There are a variety of generic and disease-specific patient reported outcome measures (PROMs) available for use in esophageal surgery. This article provides a broad overview of commonly used HRQOL instruments in esophageal surgery, including their utility in comparative effectiveness research, prognostication and shared decision-making for patients undergoing surgery for benign and malignant disorders of the esophagus.
\end{abstract}

Keywords: Esophageal surgery; esophagectomy; health-related quality of life (HRQOL); patient reported outcome measures (PROMs); patient reported outcomes (PROs); esophageal cancer

Submitted Jan 21, 2020. Accepted for publication Mar 06, 2020.

doi: $10.21037 /$ jtd.2020.03.58

View this article at: http://dx.doi.org/10.21037/jtd.2020.03.58

\section{Introduction}

Health-related quality of life (HRQOL) tools have an important place in clinical settings, providing vital information about the patient illness experience. Patient reported outcomes (PROs) are measures of HRQOL that can be quantified and used to guide therapeutic interventions, assess effectiveness of medical interventions and severity of disease (1-3). Optimizing the patient experience is a key part of health quality and therefore, is critical to delivering value-based cancer care. The PROs of esophageal surgery are also relevant (arguably, even more relevant) in benign disease as the benefit of surgery must outweigh the morbidity of the procedure, and most importantly, provide a significant improvement in baseline
HRQOL (4).

Patient reported outcome measures (PROMs) have been used in prospective studies of comparative effectiveness research involving different treatments and outcomes, and can aid in guideline development for benign and malignant esophageal disease $(1,2,5,6)$. There is also a described relationship between improved HRQOL scores and longterm survival in cancer patients (7-9). Further, using PROs to develop better prognostic estimates can inform shared decision making and further accelerate patient-centered care. There are numerous generic and disease-specific PROMs suitable for use after esophageal surgery, each with their respective advantages, disadvantages and clinical applications.

This paper will outline the available and commonly 
used instruments for assessing patient HRQOL around esophageal diseases and surgery thereof. Insight into these instruments may help readers decide about the most appropriate use of specific PROMs in the lead-up to and after benign and malignant esophageal surgery.

\section{General principles}

The extent to which HRQOL instruments are effective and useful depends on several factors, the detailed description of which is beyond the scope of this review. However, it is important to understand the intended function of an instrument as well as its performance in certain key aspects such as validity, reliability and responsiveness. Generally speaking, HRQOL surveys are designed to function as predictive, discriminative, or evaluative instruments. Predictive instruments are used to classify individuals against an external criterion and are intended for diagnostic, prognostic or screening purposes. Discriminative instruments are used to quantify differences between individuals. For example, a discriminative instrument may attempt to distinguish between patients who do and do not have gastroesophageal reflux disease (GERD); furthermore, such an instrument may also distinguish the severity of GERD between patients. Evaluative instruments are used to measure longitudinal change; for example, the instrument mentioned above may also have an evaluative component that can assess significant changes in HRQOL in one person over time and the effects of various types of treatments (10). While discriminative instruments are required only to be reliable, evaluative instruments must provide consistent measurements (i.e., reliability) and be sensitive to change (i.e., responsiveness) (11). A common method of assessing the reliability of an instrument is Cronbach's alpha. It essentially represents the correlation between the elements of an instrument (i.e., the questions in the HRQOL survey) and the extent to which these elements are measuring similar constructs and are internally consistent. Cronbach's alpha is expressed as a co-efficient between 0 and 1 ; in general, the closer the number is to 1 , the higher the correlation between constructs and thus the higher the reliability of the test. As a general rule of thumb, Cronbach's alpha coefficients of 0.7 or greater are considered to be in the acceptable range of reliability although this rule of thumb should neither be perceived as proscriptive nor universally applicable. The Cronbach's alpha values reported in this paper are derived from various studies examining the psychometric properties (i.e., reliability and validity) of the HRQOL instruments discussed.

There are numerous generic and disease-specific HRQOL surveys for use in patients undergoing esophageal surgery for benign and malignant conditions. The validity of these instruments ultimately depends on whether they measure the correct aspect of HRQOL as well as their intended function as a predictive, discriminative, or evaluative instrument (12).

\section{Available instruments}

HRQOL tools that have been previously used in evaluating patients undergoing esophageal surgery for benign and malignant diseases can be broadly grouped into diseasespecific and generic tools (Tables 1,2).

\section{Disease-specific instruments}

Many disease-specific PROMs have been developed for benign upper gastrointestinal and esophageal diseases, including GERD and Barrett's esophagus (13-15).

The Gastrointestinal Symptom Rating Scale (GSRS) is a symptom-specific instrument composed of 15 items clustered into the five symptoms of reflux, abdominal pain, indigestion, diarrhea, and constipation. While it was initially developed as a scale to measure symptoms in patients with irritable bowel syndrome and peptic ulcer disease, it has since been validated for use in patients with GERD and dyspepsia (16-18). The GSRS is reliable, with its five subscales having Cronbach's alpha coefficients between 0.61 and 0.83 (17). All five subscales of the GSRS is responsive, showing greater, statistically significant, improvements in symptom scores for treatment responders compared to nonresponders (17). The strengths of the GSRS include its ease of administration, speed (3-5-minute completion time) and its availability in multiple languages (19-24).

The GERD-Health Related Quality of Life questionnaire (GERD-HRQL) is another commonly used tool for evaluating patients with GERD undergoing surgery (25). It includes a 10-item disease-specific questionnaire addressing key symptoms, along with common side-effects of anti-reflux surgery. The GERDHRQL has excellent internal consistency and test-retest reliability, with its subscales having Cronbach's alpha coefficients between 0.89 and 0.94 and an intra-class correlation coefficient of 0.93 , respectively $(14,26)$. While it has been shown to be sensitive to treatment effects (26), 
Table 1 Commonly used patient-reported outcome instruments in esophageal surgery

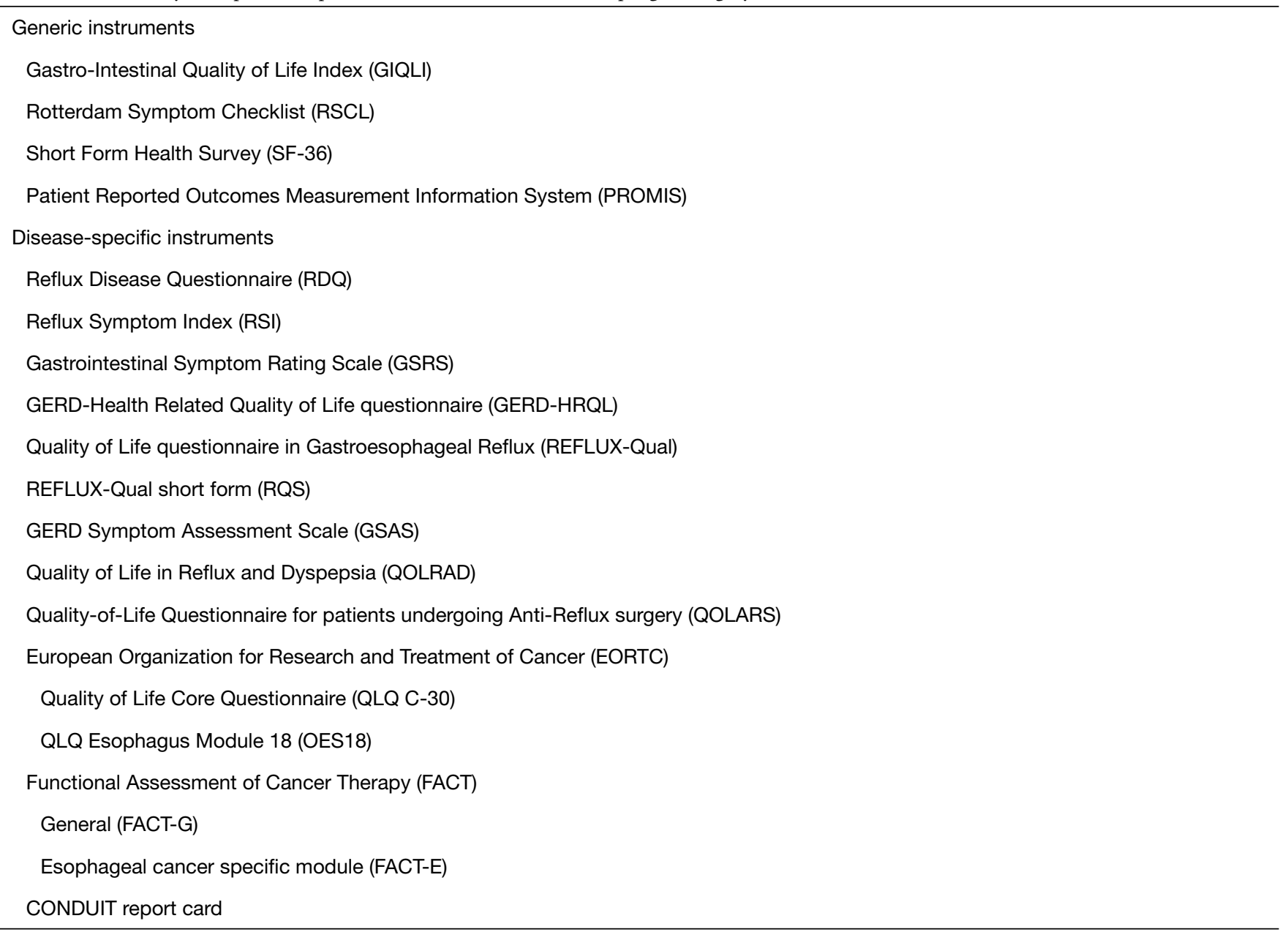

the questionnaire is limited by its inability to assess disease effects on lifestyle as well as atypical respiratory and laryngeal symptoms of GERD (27).

The Quality of Life questionnaire in Gastroesophageal Reflux (REFLUX-Qual) is a more GERD-specific questionnaire composed of 37 items within seven domains including daily activities, relationships, quality of life, mental health, worries, sleep, and appetite. The REFLUXQual was initially developed as a tool to compare patient reported outcomes between medical and laparoscopic antireflux procedures, as part of the REFLUX trial $(28,29)$. The REFLUX-Qual short form (RQS) is derived from the REFLUX-Qual and consists of eight items quantifying GERD-specific quality of life. The RQS has excellent psychometric properties, is easy to complete and is reliable $(\alpha>0.8)$ (30). The RQS also has the added benefit of being quicker to complete; as with most survey instruments, shorter version which are quicker to complete allow for reduced survey fatigue and improved survey completion rates.

The GERD Symptom Assessment Scale (GSAS) is a more comprehensive tool, composed of three separate scales that assess the burden of GERD on quality of life, the burden of symptoms, and the burden of treatment. It is one of the few tools assessing lifestyle changes, such as food avoidance, changes to activities of daily living and medications required to manage GERD (31). The GSAS has excellent internal consistency with Cronbach's alpha coefficients of 0.87 and 0.83 for its symptom and treatment scales, respectively (31). As the burden scale is not a multi-item scale, its reliability was not be assessed in this manner (31). The symptom and treatment scales also demonstrated good test-retest reliability (coefficient of 0.91 and 0.79 , respectively), however the burden scale was not as reliable 
Table 2 Characteristics of commonly used HRQOL instruments

\begin{tabular}{|c|c|c|c|}
\hline Instrument & No. of questions & Content tested & Clinical application \\
\hline $\mathrm{RDQ}$ & 12 & Physical symptoms & GERD QOL \\
\hline RSI & 9 & Physical symptoms & Reflux QOL \\
\hline GSRS & 15 (5 subscales) & Physical symptoms & Gastrointestinal symptoms QOL \\
\hline REFLUX-Qual & 37 (7 domains) & Physical symptoms mental, emotional, and social wellbeing & GERD QOL \\
\hline RQS & 8 & Physical symptoms, mental, and emotional wellbeing & GERD QOL \\
\hline GSAS & 21 (3 scales) & Physical symptom, emotional wellbeing, and lifestyle & GERD QOL \\
\hline QOLRAD & 25 (5 domains) & Physical symptoms, emotional, and social wellbeing & GERD QOL \\
\hline OES18 & 18 & Physical symptoms & Add-on to QLQ C30 for esophageal cancer \\
\hline FACT-G & 28 (4 domains) & Physical, social, emotional, functional wellbeing & Generic cancer QOL \\
\hline FACT-E & 17 & Physical symptoms & Add-on to FACT-G for esophageal cancer \\
\hline
\end{tabular}

SF-36, 36-Item Short Form Survey; RDQ, Reflux Disease Questionnaire; RSI, Reflux Symptom Index; GSRS, Gastrointestinal Symptom Rating Scale; GERD-HRQL, GERD-Health Related Quality of Life questionnaire; GERD, gastroesophageal reflux disease; REFLUX-Qual, Quality of Life questionnaire in Gastroesophageal Reflux; RQS, REFLUX-Qual short form; GSAS, GERD Symptom Assessment Scale; QOLRAD, Quality of Life in Reflux and Dyspepsia; QOLARS, Quality-of-Life Questionnaire for patients undergoing Anti-Reflux surgery; OES18, Esophagus Module 18; FACT-G, Functional Assessment of Cancer Therapy-General; FACT-E, Functional Assessment of Cancer Therapy Esophageal cancer specific module; HRQOL, health-related quality of life.

(coefficient of 0.62) (31).

The Quality of Life in Reflux and Dyspepsia (QOLRAD) questionnaire is another disease specific tool with 25 items spanning the five areas of emotions, vitality, sleep, eating/ drinking, and physical/social functioning. The QOLRAD questionnaire is validated in various languages and is a commonly used PROM before and after esophageal surgery for benign disease (19-24,32,33). The QOLRAD questionnaire has excellent internal consistency (total Cronbach's alpha coefficient of 0.97 , when assessed as a single scale), with all five domains having Cronbach's alpha coefficients between 0.89 and 0.94 (32). The Quality-ofLife Questionnaire for patients undergoing Anti-Reflux surgery (QOLARS) was also designed to assess quality of life in patients before and after anti-reflux surgery. The questionnaire was created by combining the European Organization for the Research and Treatment of Cancer Quality of Life Questionnaire (EORTC QLQ C30), modified GERD-HRQL scale and Visick scores, and was expanded upon to include questions about the patient's subjective beliefs about the efficacy of the operation and satisfaction. The QOLARS offers a comprehensive assessment of the impact of surgery on QOL, in addition to offering data in both the pre- and post-operative phases of care (34). When the GERD-specific items regarding complications and efficacy within the QOLARS questionnaire were assessed for reliability, the questionnaire demonstrated excellent internal consistency, with a Cronbach's alpha coefficient of 0.95 (34).

The most common disease-specific PROMs for use in esophageal cancer include the EORTC and Functional Assessment of Cancer Therapy (FACT) Oncologic and Organ Specific Modules $(5,6)$.

The EORTC QLQ C30 modules consist of a generic Quality of Life Core Questionnaire (QLQ C30) as well as the disease-specific Esophagus Module 18 (OES18). The QLQ C30 is a core 30-item questionnaire with a focus on daily functioning, physical and psychological symptoms, as well as impact on life activities on a four-point Likert scale $(1,2)$. As a widely used tool in different cancers, the internal consistency has been shown to be high with Cronbach's alpha coefficients $>0.70$; however, cognitive-function 
and role-related scores as well as nausea/vomiting were notable exceptions with lower reliability measures $(35,36)$. The esophageal module (OES18) includes four general categories-dysphagia, eating, reflux, and pain. There are also six additional questions regarding swallowing, choking, dry mouth, taste, coughing and speech (1). The Cronbach's alpha coefficient was found to be $>0.70$ for dysphagia and eating-related questions however, the reliability for reflux and pain symptom domains was lower (37). This limits the conclusions that can be drawn regarding the latter two symptoms.

Likewise, the Functional Assessment of Cancer TherapyGeneral (FACT-G) is used to evaluate HRQOL in many cancers. It consists of 28 questions pertaining to physical well-being, functional well-being, social/family wellbeing, and emotional well-being using a five-point Likert scale (1). This scale was developed and validated in patients with mixed cancer diagnoses, and revealed high coefficients of reliability and validity, discrimination of disease stage, and even sensitivity over time (38). Further, the esophageal cancer subscale (ECS) is a disease-specific addition to the FACT-G that explores disease-specific symptoms including two main areas of swallowing and eating, and others pertaining to xerostomia, weight loss, dysphagia and voice quality (1). When the ECS is combined with the FACT-G, the combined PROM is known as the FACT-E. This tool demonstrated very good convergent and divergent validity when compared with the EORTC QLQ30 and OES 24, and clinical variables. It was found to be reliable with a coefficient alpha $>0.70$ for all subscales and individual items (39). The FACT-E also appears to be sensitive to changes throughout the course of treatment and may aid in decision making with respect to multimodal therapy and its impact on patients (40). While comparisons of the EORTC and FACT questionnaires reveal similar categories, they are assessed differently, especially when considering social and family wellbeing, overall QOL, as well as disease-specific physical symptomatology. For instance, the social function scale differs in content when comparing the EORTC QLQ C30 and FACT-G. In addition, the disease-specific modules (i.e., the ECS in the FACT-E and the esophageal module in the QLQ C30) place different emphasis on similar domains: the FACT-E includes three questions on eating, while the EOS18 has five. These differences in scoring systems make meaningful comparisons of results difficult.

The CONDUIT report card is another PROM that is validated in patients after esophagectomy and reconstruction, primarily for carcinomas of the esophagus.
The questionnaire is composed of five multi-item scales that reflect the symptoms of dysphagia, reflux, dumping syndrome, and pain. The internal consistency was found to be $>0.70$ for all domains and with 3 of 5 domains achieving an alpha coefficient of $\geq 0.80$ (41). The CONDUIT questionnaire is particularly useful in assessing common and troublesome symptoms after esophagectomy and reconstruction that may be overlooked in other commonly used PROMs (41).

\section{Generic instruments}

Disease-specific tools may be concomitantly administered with generic tools to yield complementary information about the patient experience after surgery for benign and malignant esophageal disease.

The 36-Item Short Form Survey (SF-36) is a well-validated and widely used generic PROM. The questionnaire consists of 36 items and is grouped into eight scales: physical functioning, social functioning, role limitations caused by physical problems, role limitations caused by emotional problems, mental health, energy/ vitality, bodily pain, and general health and a single item concerning health change $(1,5)$. This results in eight scale scores and two global physical and mental component scores to reflect HRQOL $(1,5)$. The eight scales have been shown to have an alpha value exceeding 0.80 indicating high internal consistency (42) and criterion validity (43).

Recently, the Patient Reported Outcomes Measurement Information System (PROMIS) surveys aimed at globally assessing physical, mental and social health have proved to be a well-validated and useful tool for assessing HRQOL in a variety of patient populations. Currently, PROMIS offers six cancer specific tools pertaining to physical function, pain, fatigue, emotional distress, and psychosocial impact of illness $(2,44)$. The PROMIS surveys demonstrate correlation with other well-known surveys, for example the fatigue item bank had a correlation of 0.89 when compared to the SF-36 vitality score (45). Numerous other PROMs available for simultaneous use amongst patients after esophageal surgery are summarized in Tables 1,2 $(1,5,6)$.

\section{Use of PROMs for benign esophageal disease}

Surgery for benign esophageal disease is often reserved for severe and complicated presentations or medically refractory disease (46). There can be considerable overlap in the symptoms that patients with malignant and benign 
esophageal conditions experience. As such, many studies have adapted popular HRQOL scores that were initially developed in patients undergoing surgery for esophageal cancer, for use in patients with benign conditions. While this has its benefits, caution must be applied as some of the tools may focus heavily on symptoms more relevant for cancer patients and may omit pertinent symptoms that are more associated with benign esophageal conditions (41). PROs have been applied in the surgical setting for management of benign esophageal conditions and have been used in clinical studies to quantify the efficacy of different surgical and medical interventions with respect to improvements to HRQOL (47-52).

Both the GSRS and QOLRAD have been used in clinical studies to compare and quantify the effectiveness of surgical and medical interventions for GERD and Barrett's esophagus. The LOTUS Trial, for instance, examined the efficacy and HRQOL outcomes of laparoscopic antireflux surgery versus medical management in 372 patients with chronic GERD (47). The study reported that the reflux cluster within the GSRS and areas of eating/drinking and vitality in the QOLRAD questionnaire were highly sensitive and showed a greater improvement in the surgical group compared to the medical management group (47). QOLRAD has also been used as an outcome measurement in a clinical study comparing radiofrequency treatment (Stretta procedure) to laparoscopic fundoplication (LF) in patients with GERD (49). With respect to HRQOL, Stretta and LF had similar performance, and both groups showed similar and significant improvements in their posttreatment QOLRAD scores (49).

Further, Avaro et al. studied the long-term effects of redo GERD surgery, including complications, symptoms, and quality of life, in a relatively small series of 52 patients using the RQS (50). The study reported that half of the surveyed patients had high RQS scores, reflecting a good overall quality of life. RQS scores were also used to compare the performance of transthoracic and transabdominal surgery; patients that receiving transthoracic surgery reported superior quality of life at mean follow-up of 9.4 years than those receiving transabdominal surgery (50).

GERD-HRQL is a widely used PRO tool that has been used to assess quality of life outcomes in many surgical studies. In a systemic review by Hillman et al., on surgical management of proton pump inhibitor (PPI) nonresponsive GERD, GERD-HRQL was reported to be the most commonly used PRO (25). GERD-HRQL has been used in studies on LF, magnetic sphincter augment
(MSA), transoral incisionless fundoplication (TIF), and radiofrequency energy delivery studies and has successfully captured improvements to quality of life, in baseline to follow-up comparisons (25). GERD-HRQL has also been used in comparison studies on the efficacy of MSA and LF, both of which have been noted to result in comparable improvements to median GERD-HRQL scores (51). The GERD-HRQL has also been used in conjunction with the Reflux Symptom Index (RSI) and Reflux Disease Questionnaire (RDQ) to study elimination of troublesome regurgitation and extraesophageal symptoms after TIF versus PPI therapy alone (52). Elimination of troublesome symptoms was defined as scores of $<2$ on GERD-HRQL and RSI, and a reduction in frequency of moderate/severe regurgitation to less than 1 day a week, corresponding to RDQ scores of $<2$ (52). The study reported greater improvements to GERD-HRQL, RSI, and RDQ scores after TIF and concluded TIF to be more effective than PPIs for eliminating troublesome regurgitation in this patient population (52).

The GSAS is another disease-specific PROM that was developed and validated in patients undergoing LF for medically refractory GERD (31). The tool was created to address the limitations of other available tools focusing solely on disease-related symptoms and HRQOL (31). To this end, GSAS takes into consideration that the dietary, medication, and lifestyle changes that come as a result of managing GERD, do so at a cost of increased disease burden on HRQOL (31). Furthermore, the findings from this study suggests that separate symptom and treatment scales might be necessary, as they measure distinct qualities (31). For example, while some patients were not bothered by the symptoms of GERD, they reported moderate to severe burden from the treatment itself (31).

\section{Use of PROMs for esophageal carcinoma}

Carcinomas of the esophagus and gastro-esophageal junction account for the majority of esophageal resections around the world. While esophagectomy is the mainstay of curative-intent treatment for esophageal cancer, it is associated with significant treatment-related morbidity and impairments to HRQOL (4).

It has been suggested that up to $50 \%$ of patients will experience complications in the first month postoperatively (53). However, esophageal cancer also tends to be associated with poor HRQOL prior to surgical intervention $(7,40)$. It is important to recognize the 
utility of PROs in the pre-treatment phase, where poor HRQOL scores have been shown to correlate with worse overall survival $(7,8)$. In the post-operative phase, patients can go on to experience a variety of gastrointestinal and extra-intestinal symptoms related to eating, swallowing, appetite, fatigue, among others (54). Derogar and Lagergren examined HRQOL at 6 months, 3 years, and 5 years post esophagectomy using the EORTC QLQ C30 and OES18 with age and sex matched members of the general population (55). While physical function remained relatively stable in $86 \%$ of patients, $14 \%$ of patients experienced global deterioration (55). Those that survived 5 years post operatively had HRQOL comparable to the general population (55). While other factors can be implicated in the perception of health, such as developing a new baseline, PROs can be a valuable tool in assessing outcomes after surgery. For instance, Derogar et al. looked at the effect of post-operative complications, including anastomotic leak, pneumonia, return to operating room, and abscess formation on HRQOL (54). They used the QLQ C30 and the OES18 at 3 months, three and five years and found that $33 \%$ had at least one complication postoperatively (54). While global HRQOL was comparable amongst patients with and without complications, the former cohort reported more dyspnea and fatigue in the post-operative period (54).

In the era of multimodality therapy, Trudel et al. used the FACT-E subscales of physical wellbeing, functional wellbeing, and ECS to derive the Trial Outcome Index (TOI) score (40). This score is meant to assess HRQOL longitudinally, over a 36-month period, while undergoing different treatments for esophageal cancer (40). They included 84 patients who underwent chemoradiation and surgery, or chemotherapy and surgery, surgery alone, chemoradiation alone, or radiation therapy alone (40). At the 1-month assessment, all groups had a decreased HRQOL, which ultimately recovered at the 3-month mark (40). The groups which had treatment in addition to surgery had a negative impact on the HRQOL, indicating that TOI is sensitive to not only treatment initiation but also different modalities (40).

The CROSS trial also examined PROs of neoadjuvant chemoradiation (nCRT) plus surgery versus surgery alone in patients with esophageal or gastroesophageal junction cancers (56). They used specific measures in the EORTC QLQ C30 and the OES24 in the pre-treatment, preoperative and post-operative phase (3, 6, 9, and 12 months post-op) (56). Their findings suggest that although physical functioning and fatigue remain reduced after long-term follow-up, no adverse impact of nCRT is apparent on longterm HRQOL compared to patients treated with surgery alone (56).

With respect to surgical interventions, Maas et al. conducted an RCT in 2015 comparing open $v s$. minimally invasive esophagectomy (MIE) with SF-36, EORTC QLQ C30 and OES18 at baseline, 6 weeks, and 1 year after surgery (57). At 1-year mark, the MIE group reported higher HRQOL with respect to global health, pain, and physical activity (57). Similarly, Kauppila et al. carried out a systematic review of nine studies of open $v s$. MIE for cancer (58). They used the EORTC QLQ C30 and OES18 tools and found that those who underwent MIE had better outcomes at 4 to 6 weeks compared with open surgery (58). This difference in global QOL, physical function and pain was sustained at 3 months (58). By the 6-month mark, however, only physical function scores continued to differ significantly amongst the open and MIE cohorts (58).

Pre-treatment PROMs can also be used to develop prognostic estimates for patients with esophageal cancer. In a pooled, individual-patient analysis of 4 prospective studies, Kidane et al. assessed the prognostic effect of pretreatment HRQOL in patients with locally advanced esophageal cancer receiving multimodal therapy (7). After controlling for pertinent covariates including stage, surgery and age, higher pre-treatment FACT-E and ECS scores were both independently associated with better overall survival (7). Similarly, Quinten et al. conducted a review of 30 randomized controlled trials from 1986 to 2004 to examine the utility of EORTC QLQ C30 scores of patients with a variety of cancer diagnoses (59). They found that esophageal cancer was one of the only cancers in which pretreatment HRQOL was associated with overall survival (59). While these studies are not directly related to esophageal cancer patients in the modern day undergoing multimodal therapy, they highlight an important finding and potential avenue for further research.

Post-operative PROMs also appear to impact long term survival in esophageal cancer. Djärv et al. examined patients undergoing esophagectomy between 2001 and 2005 (60). The authors used nine aspects of EORTC QLQ C30 and the QLQ OES18 to classify patients according to function and symptoms (60). Patients with higher HRQOL scores at 6 months were more likely to survive to 3 and 5 years (60). In particular, patients with dysphagia or appetite loss at 6 months had the worst outcomes (60). Several other studies have created prognostic scores using combinations of 
HRQOL subscales. Chang et al. looked at patients with no residual tumor post-surgery for late death ( $>6$ months) (61). While they did not control for factors such as disease stage and tumor histology among other things, they found that low scores on the EORTC QLQ C30 in global functioning, physical, role, social, and cognitive were associated with an increased risk of death (61). Higher scores in pain, dyspnea, fatigue, insomnia, appetite loss, and financial difficulty were associated with lower survival as well (61). Similarly, certain worsened elements of the QLQ OES18 also correlated with worse survival (61). Interestingly, they did not find pre-treatment HRQOL scores to be associated with survival, however, the lack of controlling for confounding factors makes this difficult to interpret. Further research and validation in this area will help determine the clinical significance of these prognostic tools.

\section{Conclusions}

A variety of generic and disease-specific PROMs are available for use for patients undergoing esophageal surgery. Generic tools enable a broad comparison across disease states and patient populations. Generic PROMs allow for comprehensive assessment of HRQOL by capturing a range of symptoms including psychological manifestations of disease and treatment. Their use in esophageal surgery complements that of disease and organ specific tools. These scores are valuable in gleaning information around symptomology that is commonly experienced by patients with the specific condition. In addition, they can be used for comparative effectiveness research of different treatment modalities and potentially for prognostication in malignant disease.

\section{Acknowledgments}

Funding: None.

\section{Footnote}

Provenance and Peer Review: This article was commissioned by the Guest Editor (Peter Kneuertz) for the series "Patient reported Outcomes in Thoracic Surgery: A new Frontier" published in Fournal of Thoracic Disease. The article was sent for external peer review organized by the Guest Editor and the editorial office.

Conflicts of Interest: All authors have completed the ICMJE uniform disclosure form (available at http://dx.doi. org/10.21037/jtd.2020.03.58). The series "Patient reported Outcomes in Thoracic Surgery: A new Frontier" was commissioned by the editorial office without any funding or sponsorship. The authors have no other conflicts of interest to declare.

Ethical Statement: The authors are accountable for all aspects of the work in ensuring that questions related to the accuracy or integrity of any part of the work are appropriately investigated and resolved.

Open Access Statement: This is an Open Access article distributed in accordance with the Creative Commons Attribution-NonCommercial-NoDerivs 4.0 International License (CC BY-NC-ND 4.0), which permits the noncommercial replication and distribution of the article with the strict proviso that no changes or edits are made and the original work is properly cited (including links to both the formal publication through the relevant DOI and the license). See: https://creativecommons.org/licenses/by-nc-nd/4.0/.

\section{References}

1. Alghamedi A, Buduhan G, Tan L, et al. Quality of life assessment in esophagectomy patients. Ann Transl Med 2018;6:84.

2. Hirpara DH, Gupta V, Brown L, et al. Patient-reported outcomes in lung and esophageal cancer. J Thorac Dis 2019;11:S509-14.

3. Khullar OV, Fernandez FG. Patient-Reported Outcomes in Thoracic Surgery. Thorac Surg Clin 2017;27:279-90.

4. Kidane B, Coughlin S, Vogt K, et al. Preoperative chemotherapy for resectable thoracic esophageal cancer. Cochrane Database Syst Rev 2015;(5):CD001556.

5. Darling GE. Quality of life in patients with esophageal cancer. Thorac Surg Clin 2013;23:569-75.

6. Jacobs M, Macefield RC, Blazeby JM, et al. Systematic review reveals limitations of studies evaluating health-related quality of life after potentially curative treatment for esophageal cancer. Qual Life Res 2013;22:1787-803.

7. Kidane B, Sulman J, Xu W, et al. Pretreatment quality-oflife score is a better discriminator of oesophageal cancer survival than performance status. Eur J Cardiothorac Surg 2017;51:148-54.

8. Kidane B, Sulman J, Xu W, et al. Baseline measure of 
health-related quality of life (Functional Assessment of Cancer Therapy-Esophagus) is associated with overall survival in patients with esophageal cancer. J Thorac Cardiovasc Surg 2016;151:1571-80.

9. Montazeri A. Quality of life data as prognostic indicators of survival in cancer patients: an overview of the literature from 1982 to 2008. Health Qual Life Outcomes 2009;7:102.

10. Kirshner B, Guyatt G. A methodological framework for assessing health indices. J Chronic Dis 1985;38:27-36.

11. Hankins $M$. How discriminating are discriminative instruments? Health Qual Life Outcomes 2008;6:36.

12. Guyatt GH, Kirshner B, Jaeschke R. Measuring health status: What are the necessary measurement properties? J Clin Epidemiol 1992;45:1341-5.

13. Mouli VP, Ahuja V. Questionnaire based gastroesophageal reflux disease (GERD) assessment scales. Indian J Gastroenterol 2011;30:108-17.

14. Guan XL, Wang H. Quality of life scales for patients with gastroesophageal reflux disease: A literature review. Int J Nurs Sci 2015;2:110-4.

15. Crockett SD, Lippmann QK, Dellon ES, et al. Healthrelated quality of life in patients with Barrett's esophagus: a systematic review. Clin Gastroenterol Hepatol 2009;7:613-23.

16. Svedlund J, Sjödin I, Dotevall G. GSRS-A clinical rating scale for gastrointestinal symptoms in patients with irritable bowel syndrome and peptic ulcer disease. Dig Dis Sci 1988;33:129-34.

17. Revicki DA, Wood M, Wiklund I, et al. Reliability and validity of the Gastrointestinal Symptom Rating Scale in patients with gastroesophageal reflux disease. Qual Life Res 1998;7:75-83.

18. Kulich KR, Madisch A, Pacini F, et al. Reliability and validity of the Gastrointestinal Symptom Rating Scale (GSRS) and Quality of Life in Reflux and Dyspepsia (QOLRAD) questionnaire in dyspepsia: a six-country study. Health Qual Life Outcomes 2008;6:12.

19. Kulich KR, Malfertheiner P, Madisch A, et al. Psychometric validation of the German translation of the Gastrointestinal Symptom Rating Scale (GSRS) and Quality of Life in Reflux and Dyspepsia (QOLRAD) questionnaire in patients with reflux disease. Health Qual Life Outcomes. 2003;1:62.

20. Kulich KR, Calabrese C, Pacini F, et al. Psychometric validation of the italian translation of the gastrointestinal symptom-rating scale and quality of life in reflux and dyspepsia questionnaire in patients with gastro- oesophageal reflux disease. Clin Drug Investig 2004;24:205-15.

21. van Rensburg CJ, Kulich KR, Carlsson J, et al. Psychometric validation of the Afrikaans translation of two patient-reported outcomes instruments for reflux disease. South African Gastroenterology Review 2006;4:5-9.

22. Kulich KR, Regula J, Stasiewicz J, et al. Psychometric validation of the Polish translation of the Gastrointestinal Symptom Rating Scale (GSRS) and Quality of Life in Reflux and Dyspepsia (QOLRAD) Questionnaire in patients with reflux disease. Pol Arch Med Wewn 2005;113:241-9.

23. Kulich KR, Piqué JM, Vegazo O, et al. Psychometric validation of translation to Spanish of the gastrointestinal symptoms rating scale (GSRS) and quality of life in reflux and dyspepsia (QOLRAD) in patients with gastroesophageal reflux disease. Rev Clin Esp 2005;205:588-94.

24. Kulich RK, Ujszászy L, Tóth GT, et al. Psychometric validation of the Hungarian translation of the gastrointestinal symptom rating scale (GSRS) and quality of life in reflux and dyspepsia (QOLRAD) questionnaire in patients with reflux disease. Orv Hetil 2004;145:723-9, 739-44.

25. Hillman L, Yadlapati R, Whitsett M, et al. Review of antireflux procedures for proton pump inhibitor nonresponsive gastroesophageal reflux disease. Dis Esophagus 2017;30:1-14.

26. Velanovich V, Vallance SR, Gusz JR, et al. Quality of life scale for gastroesophageal reflux disease. J Am Coll Surg 1996;183:217-24.

27. Velanovich V. The development of the GERD HRQL symptom severity instrument. Dis Esophagus 2007;20:130-4.

28. Raymond JM, Marquis P, Bechade D, et al. Assessment of quality of life of patients with gastroesophageal reflux. Elaboration and validation of a specific questionnaire. Gastroenterol Clin Biol 1999;23:32.

29. Marquis P, Sapède C, Béchade D, et al. Development and Psychometric Validation of a Disease-Specific Quality of Life Questionnaire in Gastro-Oesophageal Reflux. Qual Life Res 1995;4:457-8.

30. Amouretti M, Nalet B, Robaszkiewicz M, et al. Validation of the short-form REFLUX-QUAL ® (RQS (), a gastro-esophageal reflux disease (GERD) specific quality of life questionnaire. Gastroenterol Clin Biol 2005;29:793-801.

31. Liu JY, Woloshin S, Laycock WS, et al. Symptoms and 
Treatment Burden of Gastroesophageal Reflux Disease: Validating the GERD Assessment Scales. Arch Intern Med 2004;164:2058-64.

32. Wiklund IK, Junghard O, Grace E, et al. Quality of Life in Reflux and Dyspepsia patients. Psychometric documentation of a new disease-specific questionnaire (QOLRAD). Eur J Surg Suppl 1998;(583):41-9.

33. Tofangchiha S, Razjouyan H, Nasseri-Moghaddam S. Quality Of Life in Reflux and Dyspepsia (QOLRAD) Questionnaire in Iranian Patients with GERD: A Validation Study. Middle East J Dig Dis 2010;2:84-90.

34. Zéman Z, Rozsa S, Tihanyi T, et al. Psychometric documentation of a quality-of-life questionnaire for patients undergoing antireflux surgery (QOLARS). Surg Endosc 2005;19:257-61.

35. Aaronson NK, Ahmedzai S, Bergman B, et al. The European Organization for Research and Treatment of Cancer QLQ-C30: a quality-of-life instrument for use in international clinical trials in oncology. J Natl Cancer Inst 1993;85:365-76.

36. Shih CL, Chen CH, Sheu CF, et al. Validating and Improving the Reliability of the EORTC QLQ-C30 Using a Multidimensional Rasch Model. Value Health 2013;16:848-54.

37. Blazeby JM, Conroy T, Hammerlid E, et al. Clinical and psychometric validation of an EORTC questionnaire module, the EORTC QLQ-OES18, to assess quality of life in patients with oesophageal cancer. Eur J Cancer 2003;39:1384-94.

38. Cella DF, Tulsky DS, Gray G, et al. The Functional Assessment of Cancer Therapy scale: development and validation of the general measure. J Clin Oncol 1993;11:570-9.

39. Darling G, Eton DT, Sulman J, et al. Validation of the functional assessment of cancer therapy esophageal cancer subscale. Cancer 2006;107:854-63.

40. Trudel JG, Sulman J, Atenafu EG, et al. Longitudinal Evaluation of Trial Outcome Index Scores in Patients With Esophageal Cancer. Ann Thorac Surg 2016;102:269-75.

41. Lee MK, Yost KJ, Pierson KE, et al. Patient-reported outcome domains for the esophageal CONDUIT report card: a prospective trial to establish domains. Health Qual Life Outcomes 2018;16:197.

42. Garratt AM, Ruta DA, Abdalla MI, et al. The SF36 health survey questionnaire: an outcome measure suitable for routine use within the NHS? BMJ 1993;306:1440-4.

43. Jenkinson C, Wright L, Coulter A. Criterion validity and reliability of the SF-36 in a population sample. Qual Life Res 1994;3:7-12.

44. Garcia SF, Cella D, Clauser SB, et al. Standardizing Patient-Reported Outcomes Assessment in Cancer Clinical Trials: A Patient-Reported Outcomes Measurement Information System Initiative. J Clin Oncol 2007;25:5106-12.

45. Cella D, Riley W, Stone A, et al. The Patient-Reported Outcomes Measurement Information System (PROMIS) developed and tested its first wave of adult self-reported health outcome item banks: 2005-2008. J Clin Epidemiol 2010;63:1179-94.

46. Mormando J, Barbetta A, Molena D. Esophagectomy for benign disease. J Thorac Dis 2018;10:2026-33.

47. Attwood SE, Lundell L, Hatlebakk JG, et al. Medical or surgical management of GERD patients with Barrett's esophagus: the LOTUS trial 3-year experience. J Gastrointest Surg 2008;12:1646-54; discussion 1654-5.

48. Lundell L, Miettinen P, Myrvold HE, et al. Comparison of Outcomes Twelve Years After Antireflux Surgery or Omeprazole Maintenance Therapy for Reflux Esophagitis. Clin Gastroenterol Hepatol 2009;7:1292-8.

49. Richards WO, Houston HL, Torquati A, et al. Paradigm shift in the management of gastroesophageal reflux disease. Ann Surg 2003;237:638-47; discussion 648-9.

50. Avaro JP, D'Journo XB, Trousse D, et al. Long-term results of redo gastro-esophageal reflux disease surgery. Eur J Cardiothorac Surg 2008;33:1091-5.

51. Riegler M, Schoppman S, Bonavina L, et al. Magnetic sphincter augmentation and fundoplication for GERD in clinical practice: one-year results of a multicenter, prospective observational study. Surg Endosc 2015;29:1123-9.

52. Trad KS, Barnes WE, Simoni G, et al. Transoral incisionless fundoplication effective in eliminating GERD symptoms in partial responders to proton pump inhibitor therapy at 6 months: the TEMPO Randomized Clinical Trial. Surg Innov 2015;22:26-40.

53. Viklund P, Lindblad M, Lu M, et al. Risk factors for complications after esophageal cancer resection: a prospective population-based study in Sweden. Ann Surg 2006;243:204-11.

54. Derogar M, Orsini N, Sadr-Azodi O, et al. Influence of Major Postoperative Complications on HealthRelated Quality of Life Among Long-Term Survivors of Esophageal Cancer Surgery. J Clin Oncol 2012;30:1615-9.

55. Derogar M, Lagergren P. Health-related quality of life 
among 5-year survivors of esophageal cancer surgery: a prospective population-based study. J Clin Oncol 2012;30:413-8.

56. Noordman BJ, Verdam MGE, Lagarde SM, et al. Effect of Neoadjuvant Chemoradiotherapy on Health-Related Quality of Life in Esophageal or Junctional Cancer: Results From the Randomized CROSS Trial. J Clin Oncol 2018;36:268-75.

57. Maas KW, Cuesta MA, van Berge Henegouwen MI, et al. Quality of Life and Late Complications After Minimally Invasive Compared to Open Esophagectomy: Results of a Randomized Trial. World J Surg 2015;39:1986-93.

58. Kauppila JH, Xie S, Johar A, et al. Meta-analysis of healthrelated quality of life after minimally invasive versus

Cite this article as: Ahmed M, Lau A, Hirpara DH, Kidane B. Choosing the right survey-patient reported outcomes in esophageal surgery. J Thorac Dis 2020;12(11):6902-6912. doi: $10.21037 /$ jtd.2020.03.58 open oesophagectomy for oesophageal cancer. Br J Surg 2017;104:1131-40.

59. Quinten C, Martinelli F, Coens C, et al. A global analysis of multitrial data investigating quality of life and symptoms as prognostic factors for survival in different tumor sites. Cancer 2014;120:302-11.

60. Djärv T, Lagergren P. Six-month postoperative quality of life predicts long-term survival after oesophageal cancer surgery. Eur J Cancer 2011;47:530-5.

61. Chang YL, Tsai YF, Chao YK, et al. Quality-of-life measures as predictors of post-esophagectomy survival of patients with esophageal cancer. Qual Life Res 2016;25:465-75. 